\title{
Fractal dimension of concrete mix gradation: a quantitative parameter for some concrete properties
}

\author{
Soumia Kheira Sebsadji ${ }^{1, *}$, and Kaddour Chouicha ${ }^{1}$ \\ ${ }^{1}$ LMST, Dept. of Civil Eng., USTO-MB University, BP 1505, El M'naouer, 31000 Oran, Algeria
}

\begin{abstract}
This study is based upon the fractal feature of ideal Particle Size Distributions (PSD) suggested by numerous concrete mix designs, i.e. ideal PSD can be shown to be equivalent to a power law distributions $E_{C}\left(\phi_{\mathrm{i}}\right) \propto \phi_{i}^{-D F}$, where $E_{C}\left(\phi_{\mathrm{i}}\right)$ is the number of grains of size greater than $\phi_{\mathrm{i}}$, and $D F$ is a nonwhole number called fractal dimension. This fact allows us to analyze the solid skeleton of a concrete mix (all solid components of the mixture) as a fractal structure, thus to determine some of physical properties of the concrete mixture. For $D F$ ranging from 2.5 to 3, and based upon many parameters of the concrete mix (as the granular range, the volumetric fraction of solids in the concrete mixture...), analytical formulas have been proposed relating $D F$ and some properties of the concrete in the solid state. The required properties are the coarse-to-fine aggregate ratio, the fineness modulus of the sand fraction, the average grain size and volume of the fine fraction in the concrete mix. The focus of this research is to develop formulas by which concrete properties can be predicted knowing the concrete mix gradation, i.e. the mix design method used.
\end{abstract}

\section{Introduction}

Fractals can be defined as disordered systems. One of the main properties of fractals is their power law behavior of the form: $N(L>r) \propto r^{-D F}$, such as $N$ is the number of objects in the system with size greater than $r, D F$ is a non-integer number referred to as Fractal Dimension and the symbol ' $\propto$ ' stands for 'proportional to' [1, 2].

Concrete mixtures can be considered as fractal objects because their corresponding solid particle size distributions (PSD), since they must be as close as possible to one of the ideal grading curves, they can be transformed to "grain number $E_{C}$ " vs "grain size $\phi_{i}$ " distributions, following a fractal power law of type $E_{C} \propto \phi_{i}^{-D F}[1,3,4]$.

To get a better understanding of this, we give an example. First, we transform an ideal grading curve according to Fuller \& Thompson [5], on fitted straight line $E_{C}\left(\phi \geq \phi_{\mathrm{i}}\right)=f\left(\phi_{\mathrm{i}}\right)$ in log-log scale (see Fig. 1). The data points will be obtained by the following expressions:

$$
E_{C}\left(\phi \geq \phi_{i}\right)=\sum_{j=1}^{i} E_{j}
$$

Such as:

$$
E_{j}=\frac{1}{\rho v_{j}} R_{j} \text { and } v_{j}=\frac{\pi}{6} \phi_{j}^{3}
$$

Where $E_{C}\left(\phi \geq \phi_{\mathrm{i}}\right)$ denotes the grain number of size greater than $\phi_{i} ; E_{j}$ the grain number of one size class $\phi_{j} ; R_{j}$ the weight residue on sieve $\phi_{j}$ mesh; $v_{j}$ the volume per grain of size $\phi_{j}$ and $\rho$ the mass density of aggregates. In Fig. 1, using linear regression, fitting results show a good linear fit between $E_{C}\left(\phi \geq \phi_{\mathrm{i}}\right)$ and $\phi_{i}$ $\left(R^{2} \approx 1\right)$. The equation of the best linear fit is:

$$
\begin{gathered}
\log E_{C}\left(\phi_{\mathrm{i}}\right)=a+(-D F) \log \phi_{\mathrm{i}} \\
\Rightarrow E_{C}\left(\phi_{\mathrm{i}}\right)=\mathcal{C} \phi_{i}^{-D F}
\end{gathered}
$$

$\mathcal{C}=10^{a}$ is a parameter depending on some properties of concrete [3], the slope of the best-fitted line representing the relationship between $E_{C}$ and $\phi_{i}$ is the fractal dimension $D F$.

One can achieve same results whatever the method of concrete composition (see $[1,3,4]$ ), that enables us to assume PSDs of real concrete mixtures as fractals, which allows identifying them by knowing two parameters, $D F$ value and the total particle size range $d / D$.

\section{Grading curve of a fractal PSD}

The solid concrete mixes will be noted $\mathcal{M}_{d / D}^{D F}$, meaning that the grain size distribution of the mix is fractal and that the grain size is ranging from $d$ to $D$.

Each mixture will be constituted by particles of $n$ monosized classes, numbered from $\phi_{1}$ for the largest to $\phi_{\mathrm{n}}$ for the smallest ones (according to a geometric progression of reason $\sqrt[10]{10}$ ). Such as grains belong to a class ' $i$ ' will be the set of particles of number $E_{i}$,

*Corresponding author: soumia.sebsadji@univ-usto.dz 

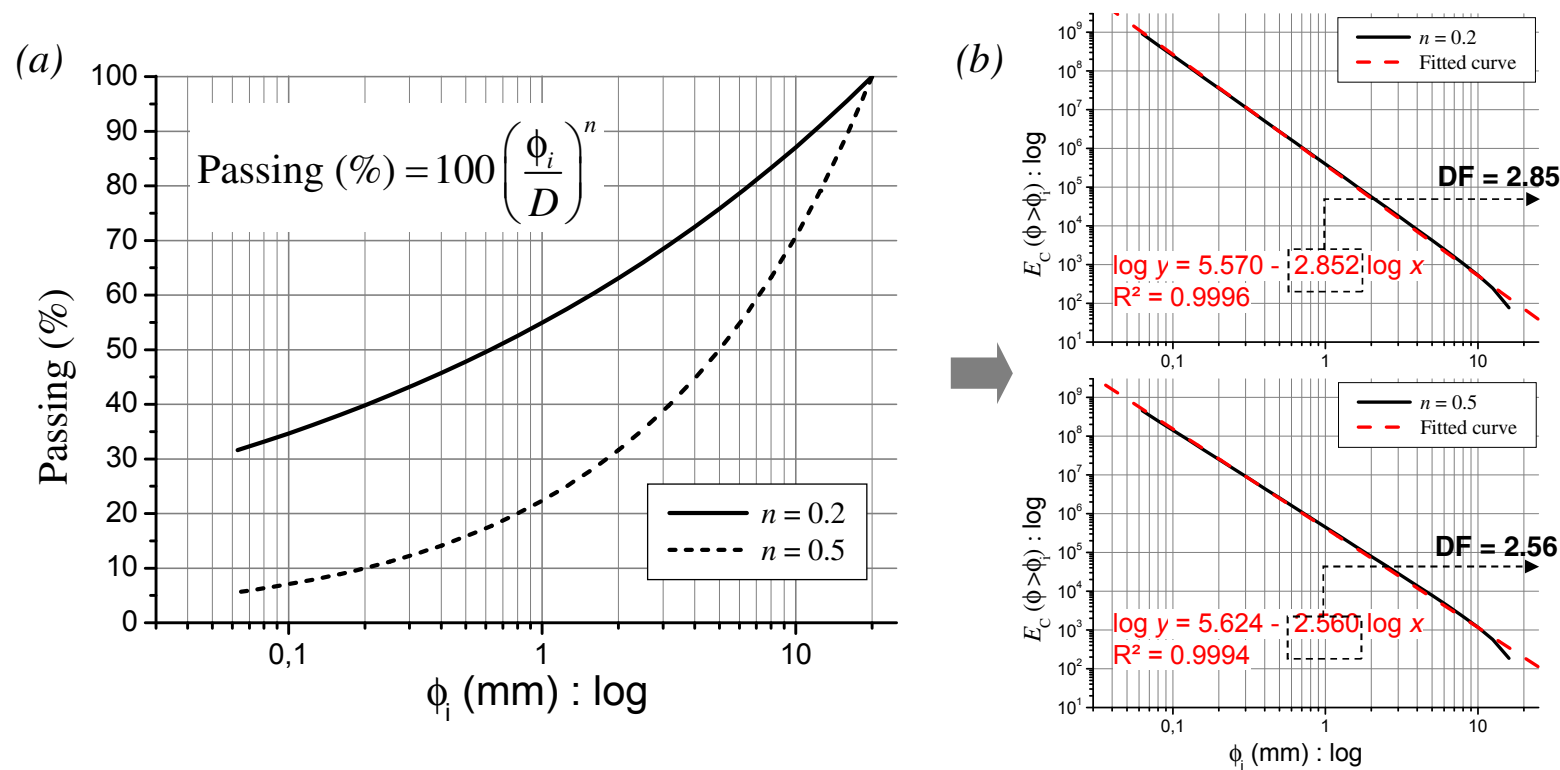

Fig. 1. (a) Fuller-Thompson ideal grading curve $(b)$ The corresponding fitted curves: $E_{C}\left(\phi \geq \phi_{\mathrm{i}}\right)=f\left(\phi_{\mathrm{i}}\right)$

retained between two successive sieves with a mesh size of $\phi_{\mathrm{i}}$ and $\phi_{\mathrm{i}+1}$, as shown in the following expressions:

$$
\forall i \in[1, n]: \phi_{i}>\phi_{i+1} ; \quad \text { and }: \frac{\phi_{i}}{\phi_{i+1}}=\lambda=\sqrt[10]{10}
$$

Such as $\phi_{1}=D ; \phi_{n}=d$. From the above relations, we can also obtain:

$$
\phi_{2}=\frac{\phi_{1}}{\lambda}=\frac{D}{\lambda} ; \phi_{3}=\frac{\phi_{2}}{\lambda}=\frac{D}{\lambda^{2}} \ldots \Rightarrow \phi_{i}=\frac{D}{\lambda^{i-1}}
$$

From Eq. (3), the grain number of grains of size greater than $\phi_{i}$ is given as: $E_{C}\left(\phi_{i}\right)=\mathcal{C} \phi_{i}^{-D F}$, thus $E_{i}$ the grain number of one size class $\phi_{i}$ can be obtained as follows:

$$
E_{i}= \begin{cases}\mathcal{C} \phi_{i}^{-D F}, & \text { if } i=1 \\ \mathcal{C}\left(\phi_{i}^{-D F}-\phi_{i-1}^{-D F}\right), & \forall i \in[2, n]\end{cases}
$$

By replacing $\phi_{i}=\frac{D}{\lambda^{i-1}}$, in the above equation, we obtain:

$$
E_{i}=C D^{-D F} \begin{cases}1, & \text { if } i=1 \\ \left(\lambda^{D F(i-2)}\right)\left(\lambda^{D F}-1\right), & \forall i \in[2, n]\end{cases}
$$

Based on these equations, one can obtain the grading curve of a fractal granular mixture $R_{C}\left(\phi_{i}\right)=f\left(\phi_{i}\right)$ or $T_{C}\left(\phi_{i}\right)=f\left(\phi_{i}\right)$ (resp. \% cumulative retained or passing), obtained by transformation of the fractal straight line $E_{C}\left(\phi_{i}\right)=f\left(\phi_{i}\right)$. First, the percentage of material retained $R_{i}(\%)$ on mesh sieve $\phi_{i}$ can be expressed as:

$$
R_{i}(\%)=100 \frac{R_{i}}{\sum_{j=1}^{n} R_{j}}
$$

On another hand, the weight retained on mesh sieve $\phi_{i}$, noted $R_{i}$, can be expressed as follows:

$$
R_{i}=E_{i} m_{i}=E_{i}\left(\rho v_{i}\right)
$$

Where: $\left\{\begin{array}{l}E_{i}: \text { number of grains in size class } \phi_{i} \\ m_{i}: \text { mass of an individual grain of size } \phi_{i}\end{array}\right.$.

Developments of the above equations yields to the equations of a fractal grading curves (for further details see reference [6]):

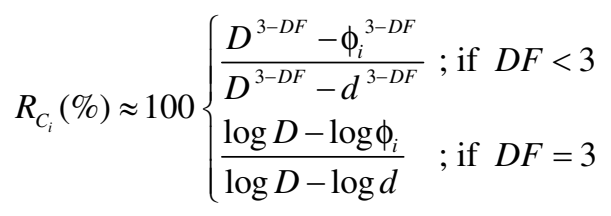

\section{Physical properties of concrete}

Using equations in (10) we can determine grading curves for some granular mixes (dry state) $\mathcal{M}_{d / D}^{D F}$. That will enable us to determine some physical properties of concrete mixes. The fractal dimension considered in these mixes will be in the range: $2.5 \leq D F \leq 3[4,6]$.

\subsection{Ratio of fine-to-coarse fractions}

The ratio of fine-to-coarse fractions of the dry concrete mix, noted $G / F$, can be determined by the use of the following expression, where $\phi_{G F}$ represents the particle size cut between fine and coarse fractions:

$$
\begin{aligned}
G / F & =\frac{\text { weight of coarse fraction }\left(\phi_{i}>\phi_{G F}\right)}{\text { weight of fine fraction }\left(\phi_{i} \leq \phi_{G F}\right)} \\
& =\frac{M_{T} R_{C_{G F}}(\%)}{M_{T}\left[100-R_{C_{G F}}(\%)\right]}=\frac{R_{C_{G F}}(\%)}{T_{C_{G F}}(\%)}
\end{aligned}
$$


By replacing $R_{C_{i}}(\%)$ (Eq. (10)); Eq. (11) can be rewritten as follows, such as $d<\phi_{G F}<D$ :

$$
G / F=\left\{\begin{array}{l}
\frac{D^{3-D F}-\phi_{G F}^{3-D F}}{\phi_{G F}^{3-D F}-d^{3-D F}} ; \text { if } D F<3 \\
\frac{\log D-\log \phi_{G F}}{\log \phi_{G F}-\log d} ; \text { if } D F=3
\end{array}\right.
$$

Fig. 2 illustrates an example of calculation of the ratio $G / F$ for some dry concrete mixes, by using Eqs in (12). In this example, variables are $d, D$ and $D F$.

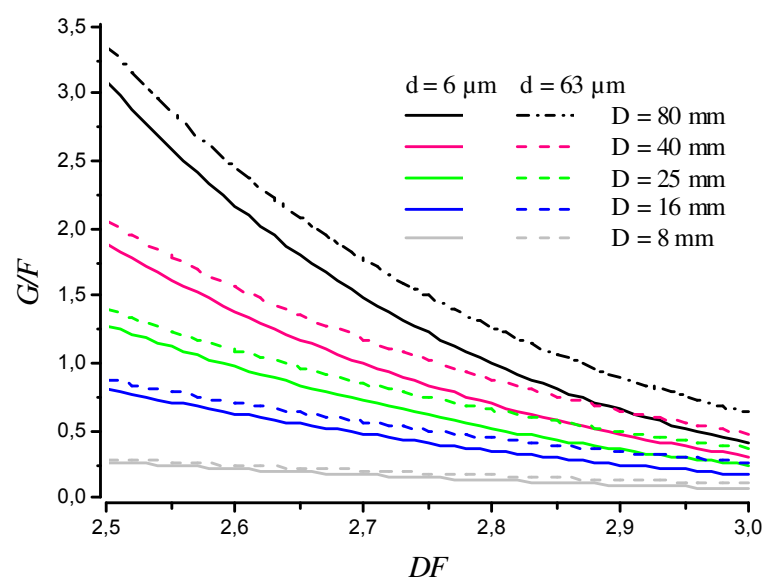

Fig. 2. $G / F$ ratio related to $d, D$ and $D F\left(\phi_{G F}=5 \mathrm{~mm}\right)$

\subsection{Average diameter of grains}

Characteristic diameters of grains as the average grain diameter are relevant parameters involved in the description of size distribution of granular mixtures. The average particle diameter, noted $D_{50}$, is defined as the size for which $50 \%$ of the material of larger particles, i.e. the cumulative weight percent of material passing a sieve size of $D_{50}$ noted $P_{50 \%}$ is $50 \%$ [7]. According to this definition, the size $D_{50}$ of a dry concrete mix $\mathcal{M}_{d / D}^{D F}$, can be expressed as follows (by using Eqs in (10)):

$$
P_{50 \%}=100\left\{\begin{array}{l}
\frac{D_{50}^{3-D F}-d^{3-D F}}{D^{3-D F}-d^{3-D F}} ; \text { if } D F<3 \\
\frac{\log D_{50}-\log d}{\log D-\log d} ; \text { if } D F=3
\end{array}\right.
$$

From which we can obtain $D_{50}$ in simplified form as follows, such as $P_{50}=\frac{1}{100} P_{50 \%}$ and $\overline{P_{50}}=1-P_{50}$ :

$$
D_{50}= \begin{cases}\left(P_{50} D^{3-D F}+\overline{P_{50}} d^{3-D F}\right)^{\frac{1}{3-D F}} & ; \text { if } D F<3 \\ D^{P_{50}} d^{\overline{P_{50}}} & \text {; if } D F=3\end{cases}
$$

Fig. 3 illustrates an example of calculation of $D_{50}$ for some dry concrete mixes by using Eqs in (14). In this example, considered variables are $d, D$ and $D F$.

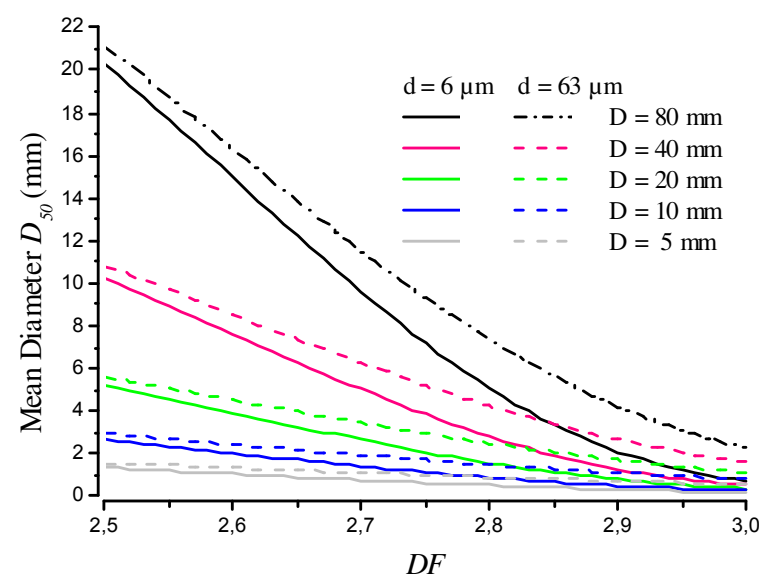

Fig. 3. Mean diameter $D_{50}$ related to $d, D$ and $D F$

\subsection{Amount of fines in a concrete mix}

Fines are beneficial in a concrete mix, because they fill voids, reduce cement and improve workability [8-10]. Fines in the concrete mix must fill the voids of the bigger aggregates; hence, the optimum content of fines is related to the granular extent $d / D$ and to the PSD of the mix, i.e. $D F$.

In this work, the amount of fines will be considered consisting of aggregate fines, mineral addition(s) and cement with most particles passing through $63 \mu \mathrm{m}$ sieve, this sieve opening will be denoted $\phi_{\text {fines }}$. In this section, we will derive equations to determine the amount of fines $\left(\leq \phi_{\text {fines }}\right)$, noted $A_{\text {fines }}$ (in $\%$ ), in a granular mix $\mathcal{M}_{d / D}^{D F}$, as follows:

$$
A_{\text {fines }}(\%)=100\left\{\begin{array}{l}
\frac{\left(\phi_{\text {fines }} / d\right)^{3-D F}-1}{(D / d)^{3-D F}-1} ; \text { if } D F<3 \\
\frac{\log \left(\phi_{\text {fines }} / d\right)}{\log (D / d)} \quad \text { if } D F=3
\end{array}\right.
$$

$M_{T}$ is the total mass of the concrete ingredients (except water), hence $M_{\text {fines }}$, the mass of fines in this mix that can be composed of sand fines $\left(G_{1}\right)$, cement $\left(G_{2}\right)$ and mineral addition $\left(G_{3}\right)$, can be given as follows:

$$
\begin{aligned}
M_{\text {fines }} & =M_{T} \frac{A_{\text {fines }}(\%)}{100} \\
& =G_{1}+G_{2}+G_{3}=M_{T}\left(P_{1}+P_{2}+P_{3}\right)
\end{aligned}
$$

Such as $P_{1}, P_{2} \& P_{3}$ are the volumetric proportions of fines and $P_{1}+P_{2}+P_{3}=1$. By using Eq. (16), we can determine the fines content, denoted $V_{\text {fines }}$, as follows:

$$
V_{\text {fines }}=\frac{G_{1}}{\rho_{G 1}}+\frac{G_{2}}{\rho_{G 2}}+\frac{C}{\rho_{C}}
$$



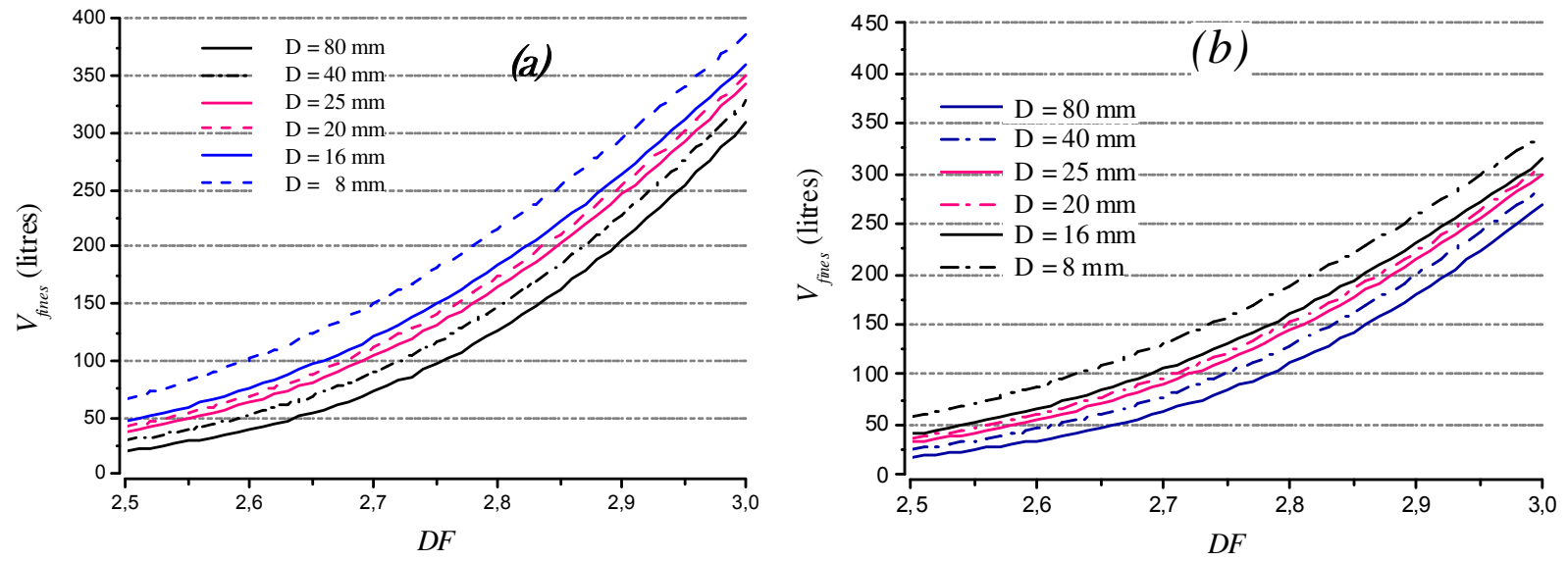

Fig. 4. Amount of fines of concrete mixes, such as $M_{T}=2350 \mathrm{~kg} / 1 \mathrm{~m}^{3}$ of concrete : (a) Fines $=100 \%$ fines of aggregates $\left(\rho_{G}=2.5 \mathrm{~g} / \mathrm{cm}^{3}\right)(b)$ Fines $=100 \%$ cement $\left(\rho_{C}=3.2 \mathrm{~g} / \mathrm{cm}^{3}\right)$.

$\rho_{G 1}, \rho_{G 2} \& \rho_{C}$ are respectively the absolute densities of each type of fines in concrete: aggregates, mineral addition and cement. By replacing Eq. (16) in (17), we obtain:

$$
V_{\text {fines }}=M_{T} \frac{A_{\text {fines }}(\%)}{100}\left(\frac{P_{1}}{\rho_{G 1}}+\frac{P_{2}}{\rho_{G 2}}+\frac{P_{3}}{\rho_{C}}\right)
$$

However, for $m$ types of concrete fines, of the absolute densities: $\rho_{1}, \ldots, \rho_{m} ; V_{\text {fines }}$ can be expressed as follows:

$$
V_{\text {fines }}=M_{T} \frac{A_{\text {fines }}(\%)}{100}\left(\frac{P_{1}}{\rho_{1}}+\frac{P_{2}}{\rho_{2}}+\ldots+\frac{P_{m}}{\rho_{m}}\right)
$$

The mean density of all concrete fines $\rho_{\text {fines }}$, can be expressed as follows:

$$
\rho_{\text {fines }}=\frac{M_{\text {fines }}}{V_{\text {fines }}}=\frac{1}{\frac{P_{1}}{\rho_{1}}+\frac{P_{2}}{\rho_{2}}+\ldots+\frac{P_{m}}{\rho_{m}}}
$$

By combining Eqs (15)-(20), we obtain the following equations, allowing the determination of the amount of fines by unit mass of concrete mix:

$$
V_{\text {fines }}=\frac{M_{\text {fines }}}{\rho_{\text {fines }}}=\frac{P M_{T}}{100} \begin{cases}\frac{\left(\phi_{\text {fines }} / d\right)^{3-D F}-1}{(D / d)^{3-D F}-1} & \text {; if } D F<3 \\ \frac{\log \left(\phi_{\text {fines }} / d\right)}{\log (D / d)} & \text {; if } D F=3\end{cases}
$$

Such that: $P=\frac{P_{1}}{\rho_{1}}+\frac{P_{2}}{\rho_{2}}+\ldots+\frac{P_{m}}{\rho_{m}}$

Fig. 4 illustrates graphically the obtained results of the calculation of the amount of fines for some dry concrete mixes by using Eqs in (21). In this example, the variables are $D$ and $D F$.

\subsection{Fineness modulus of sand fraction}

The fineness modulus $(F M)$ is an empirical factor used to estimate the proportions of fine and coarse aggregates in concrete mixtures. According to NF P18-541 standard [11], $F M$ can be obtained by adding the cumulative percentages of aggregate retained on each of the standard sieves ranging from 80 to $0.16 \mathrm{~mm}$ and dividing this sum by 100 . Generally, $F M$ of sand shall not be less than 1.8 or more than 3.2 to make good concrete.

According to the above definition, $F M$ of the sand fraction $\left(\phi_{m}<\phi_{i}<\phi_{\text {sand }}\right)$ in a granular mix $\mathcal{M}_{d / D}^{D F}$, can be written as follow:

$$
\begin{array}{r}
F M=\frac{1}{100} \sum_{i=1}^{t} \bar{R}_{C}\left(\begin{array}{c}
\phi_{i} \\
F M
\end{array}\right) \\
\text { and: } \phi_{F M} \in\left\{\begin{array}{l}
\phi_{m} / \phi_{\text {sand }} \\
{\left[\begin{array}{c}
F M_{j}
\end{array}\right]}
\end{array}\right.
\end{array}
$$

Such as: $\left[F M_{j}\right]=[0.16 ; \ldots ; 80 \mathrm{~mm}]=$ sieves considered in $F M$ definition; $t=$ number of sieves $\phi_{i} ; \bar{R}_{C}\left(\phi_{k}\right)$ the cumulative $\%$ passing of the sand fraction in the mix given by Eq. (24):

$$
\bar{R}_{C}\left(\phi_{k}\right)=100 \begin{cases}\frac{\phi_{s a n d}^{3-D F}-\phi_{k}^{3-D F}}{\phi_{s a n d}^{3-D F}-\phi_{m}^{3-D F}} & \text {;if } D F<3 \\ \frac{\log \phi_{s a n d}-\log \phi_{k}}{\log \phi_{s a n d}-\log \phi_{m}} & \text {;if } D F=3\end{cases}
$$

By substituting the above expressions in Eq. (22), we obtain the following expressions allowing the determination of $F M$ :

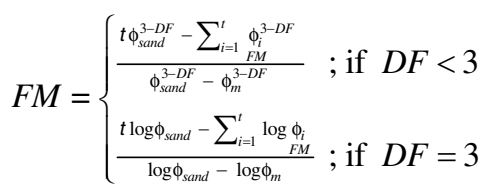

Fig. 5 illustrates an example of calculation of $F M$ for some dry concrete mixes by using Eqs in (24). In this example, the considered variables are $D F$ and the sand fraction sizes: $\phi_{\text {sand }}$ and $\phi_{m}$. 


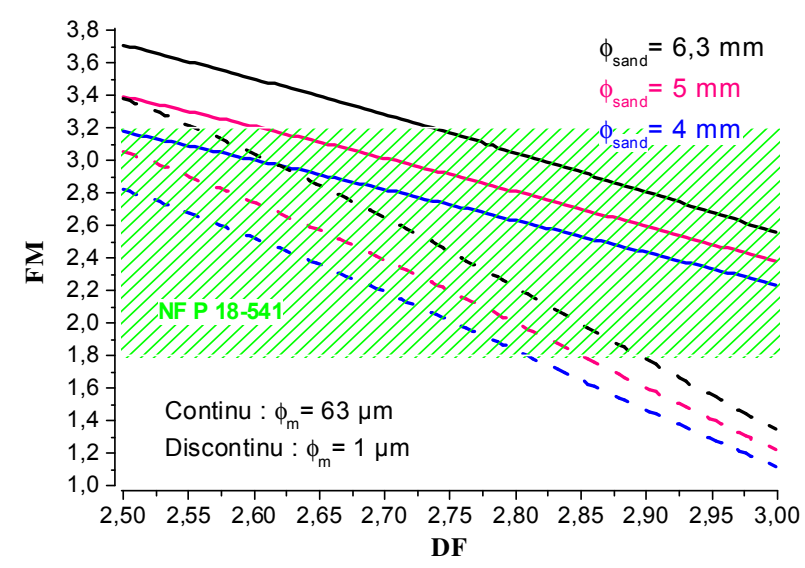

Fig. 5. $F M$ of sand fraction of granular mixes, related to $D F$ and the limit sizes $\phi_{\text {sand }} \& \phi_{m}$. In green, recommended $F M$ values $(1.8 \leq F M \leq 3.2)$ according to NF P $18-541$ standard.

\section{Analysis and conclusion}

The key point of this study is to consider ideal size gradations for concrete, hence concrete mix gradations as almost fractal. This allowed us first, to propose a general fractal gradation curve, which would describe any concrete mix gradation with the help of two parameters: the granular extent $d / D$ and the fractal dimension $D F$. Second, to propose simple analytical formulas allowing the calculation of some physical properties of the dry concrete mixes.

According to our analytical results, $D F$ values varying from 2.5 to 3 indicate that the concrete mix gradation is coarser for small $D F$ values and finer for more $D F$ values, thus we can see decreasing $G / F$ ratio, fineness modulus $F M$ and average particle size $D_{50}$; and a significant increase of the amount of fines $V_{\text {fines }}$ in the concrete mix. These results are in agreement with others in the literature, for instance, some researchers have proposed an optimal gradation curve $\%$ Passing $=100\left(\frac{\phi_{i}}{D}\right)^{q}$, such as $q=0.37$ (corresponding to $D F=2.63$ ) to achieve maximum compactness for ordinary concrete and $q=0.30$ (corresponding to $D F=2.70$ ) for Self-Compacting Concrete that the mix must be finer [12].

Therefore, the fractal dimension can be a good characterization parameter to be adjusted to obtain some required properties of concrete.

\section{References}

1. A. Lecomte, A. Thomas, Mater. Struct., 25, 10 (1992)

2. Z. Wang, Q. Cheng, L. Cao, Q. Xia, Z. Chen, Math. Geol., 39, 16 (2007)

3. K. Chouicha, Mater. Struct., 39, 17 (2006)
4. S. K. Sebsadji, K. Chouicha, Int. J. Solids Struct., 49, 10 (2012)

5. A. Amirjanov, K. Sobolev, Part. Sci. Technol., 26, 16 (2008)

6. S. K. Sebsadji, The use of fractal analysis for assessing concrete properties, Doctoral thesis, USTO-MB, Algeria (2013)

7. C. Paderno, Comportement du ballast sous l'action $d u$ bourrage et du trafic ferroviaire, Doctoral thesis, EPFL, Switzerland (2010)

8. J. Baron, J. Ollivier, Les Bétons : Bases et données pour leur formulation, Eyrolles Ed., Paris, (1996)

9. F. de Larrard, Structures granulaires et formulation des bétons, LCPC, Paris (2000)

10. G. Dreux, J. Festa, Nouveau guide du béton et de ses constituants, Eyroles Ed.. Paris (2007)

11. NF P18-541, Granulats, Granulats pour béton hydrauliques, AFNOR, France (1994)

12. H. He, Computational Modelling of Particle Packing in Concrete, Doctoral thesis, Delft University of Technology, The Netherlands (2010) 